\title{
Alcohol-related admissions to an intensive care unit in Dublin
}

\author{
M. McKenny $\cdot$ S. O'Beirne $\cdot$ C. Fagan • \\ M. O'Connell
}

Received: 14 September 2009/Accepted: 4 May 2010/Published online: 28 May 2010

(C) Royal Academy of Medicine in Ireland 2010

\begin{abstract}
Background Excessive alcohol consumption is ingrained in Irish society and is known to have significant adverse health consequences, including adverse outcomes for critically ill patients. Previous assessments of alcohol-related intensive care unit (ICU) admissions were felt to have underestimated the scale of this problem.

Aims A study was designed to objectively measure alcohol-related ICU workload.

Methods We prospectively recorded the number of patients who were admitted to St James's Hospital ICU as a result of alcohol misuse during a 6-month period in 2008. Admission diagnosis, Acute physiology and Chronic Health Evaluation Score 2 (APACHE 2), ICU length of stay and 30-day mortality were recorded.

Results The study group occupied $16.7 \%$ of the total available ICU bed-days, experienced longer stays and higher mortality.

Conclusion This study adds to the data available on the scale of alcohol-related problems amongst Irish healthcare system patients. Prioritisation of primary preventative strategies is necessary.
\end{abstract}

Keywords Alcohol misuse - Intensive care unit . Mortality $\cdot$ Length of stay $\cdot$ Bed-days

M. McKenny $(\varangle) \cdot$ S. O’Beirne $\cdot$ C. Fagan $\cdot$ M. O'Connell

Department of Anaesthesia and Intensive Care,

St James's Hospital, Dublin 6, Ireland

e-mail: mikemckenny@eircom.net

\section{Introduction}

Alcohol is the third most important risk factor for premature death and ill health in the European Union [1]. It has a multi-system effect; excessive consumption contributes to negative outcomes for critically ill patients and is implicated in the pathogenesis of more than 60 diseases [2]-[6]. Additionally, alcohol misuse is associated with a variety of other harms including accidents, trauma, child neglect, relationship difficulties, public safety problems and productivity loss [1].

On a per capita basis, the Irish population are amongst the heaviest consumers of alcohol in Europe [7]. The estimated cost to the Irish economy of alcohol-related harm in 2003 was $€ 2.65$ billion [8]. Significant health-care resources are consumed; 841,161 in-patient bed-days in Irish hospitals in the 10-year period to 2004 were occupied due to alcohol-related problems [9]. Recent evidence shows that the mortality rate due to alcohol-related illness and harm is higher than previously estimated and is thought to be responsible for $4.4 \%$ of all deaths in the Republic [10].

Anecdotal reports suggest that a significant but previously unmeasured proportion of ICU workload in Ireland is consequent on alcohol misuse. In 2008 the health service executive (HSE) estimated that $10 \%$ of Intensive Care Unit (ICU) admissions were alcohol-related [1]. This study was designed to objectively quantify the workload at St James's Hospital ICU which was the direct result of alcohol misuse.

\section{Methods}

St James's Hospital has a total of 901 beds and a catchment area that includes a large part of inner-city Dublin [11]. It is 
a tertiary referral centre for a wide range of medical and surgical specialities. The 15 -bed general ICU provides care for adult patients from all specialities within the hospital, and for patients with head and brain injuries.

A prospective audit was conducted from 1st June to 30th November 2008, during which time the number of patients whose ICU admission resulted directly from alcohol misuse was recorded. The patient's admission was regarded as being causally related to alcohol misuse if excessive alcohol consumption led to one or more of the following admission diagnoses: (1) alcohol withdrawal syndrome; (2) alcoholic cirrhosis with hepatic failure and/or upper gastrointestinal tract bleeding secondary to portal hypertension; (3) alcoholic pancreatitis; (4) alcoholic hepatitis; (5) trauma secondary to alcohol intoxication; (6) medical condition secondary to alcohol intoxication such as aspiration pneumonia. The diagnosis was made by the admitting ICU consultant. For each patient who met the study inclusion criteria, the following parameters were recorded: age, gender, length of ICU stay in days, APACHE 2 score, 30-day mortality.

\section{Results}

There were a total of 275 admissions to the ICU during the study period. Thirty-three patients met the study inclusion criteria and their admission diagnoses are shown in Table 1 . The study group comprised $12 \%$ of total admissions, $19 \%$ of emergency admissions, and occupied $16.7 \%$ of the total available bed-days during the study period. All study group patients had unplanned admissions.

The average ICU length of stay of a patient meeting the study inclusion criteria was 12.3 days, which was approximately double the average stay of a non-study group patient who was in the ICU during this 6-month period. The study group comprised predominantly male patients (88\%). Average APACHE 2 score was 17.7. Thirty day mortality for the study group was $24.4 \%$, compared to a non-study group mortality of $19 \%$. The causes of death for the study group are displayed in Table 2 . The cost of

Table 1 Number of patients and admission diagnoses

\begin{tabular}{lclc}
\hline Admission diagnosis & Male & Female & Total \\
\hline Alcoholic cirrhosis & 11 & 1 & 12 \\
Coma, alcohol intoxication & 5 & 1 & 6 \\
Medical condition due to alcohol & 4 & 1 & 5 \\
Trauma due to alcohol intoxication & 3 & 1 & 4 \\
Alcohol withdrawal seizures & 3 & 0 & 3 \\
Pancreatitis due to alcohol & 3 & 0 & 3 \\
\hline
\end{tabular}

Table 2 Cause of death of study group patients

\begin{tabular}{ll}
\hline Cause of death & Number of patients \\
\hline Decompensated hepatic failure & 5 \\
Sepsis and multi-organ failure & 2 \\
Hypoxic encephalopathy & 1 \\
\hline
\end{tabular}

looking after the patients meeting the study inclusion criteria in the ICU was in the region of $€ 1$ million.

\section{Discussion}

All of the patients in the study group were admitted on an emergency basis which tends to create extra workload at ward level and for bed management services. The patients with an alcohol-related diagnosis tended to have a longer than average stay compared to non-study group patients, a factor which is independently associated with increased resource consumption. Study group patients also experienced a higher mortality rate.

Similar studies have been published. A Finnish prospective study conducted in a 15-bed adult tertiary referral ICU over 12 months in 2002-2003 identified a definite causal relationship between alcohol misuse and diagnosis in $17.5 \%$ of admissions, which accounted for $17.8 \%$ of total available bed-days [12]. Interestingly, the average length of ICU stay was 1.2 days, a much shorter time than in our study (12.3 days). The authors of the Finnish study felt that this short length of stay was due to the large proportion of patients with gastrointestinal bleed. In our study, the patients tended to have conditions such as pancreatitis, trauma and hepatic failure which required longer admissions. A prospective audit in a tertiary referral adult ICU in Northern Ireland carried out over 5 months in 2006-2007 identified $9.43 \%$ of admissions as being causally related to alcohol misuse [13]. These patients occupied $13.9 \%$ of total available bed-days and had a median stay of 10 days; a large proportion of these patients had trauma. In the Belfast study, the ICU and hospital mortality rates were 23.3 and $30 \%$, respectively, which broadly accords with our 30 -day mortality rate of $24.4 \%$. In relation to the percentage of total available ICU bed-days occupied as a result of alcohol misuse, the figures in this study are broadly in line with the international experience (Table 3).

Alcohol-related illness places a heavy burden on healthcare systems. The published evidence shows that patients who consume alcohol excessively experience greater morbidity across a range of outcomes. They are more likely to develop respiratory failure, require ICU admission and to die as a result of a community acquired pneumonia [2]. They are more likely to develop a nosocomial ventilator associated 
Table 3 Alcohol-related ICU admissions; summary of published data

\begin{tabular}{lll}
\hline Type of ICU and location & $\begin{array}{l}\% \text { admissions } \\
\text { due to alcohol }\end{array}$ & $\begin{array}{l}\% \text { of total bed-days } \\
\text { due to alcohol }\end{array}$ \\
\hline $\begin{array}{l}\text { Mixed ICU, Finland, 2002 } \\
\text { [12] }\end{array}$ & 17.5 & 18 \\
$\begin{array}{l}\text { Mixed ICU, Belfast, 2006 } \\
\quad \text { 13] }\end{array}$ & 9.4 & 14 \\
$\begin{array}{l}\text { Mixed ICU, St James's, } \\
\text { Dublin, 2008 }\end{array}$ & 12 & 16.7 \\
\hline
\end{tabular}

pneumonia [3]. The general ICU patient who consumes alcohol excessively is twice as likely to develop acute respiratory distress syndrome (ARDS) as matched controls [4]. Alcohol abuse was found to be independently associated with sepsis, septic shock and increased mortality in a retrospective cohort study of 12,000 ICU patients in Denver [5]. Elective surgical patients with a current history of alcohol abuse experience a higher incidence of peri-operative complications including respiratory failure, bleeding requiring transfusion, delayed wound healing and longer hospital stay [14]. In one Spanish study, elective surgical patients undergoing a variety of minor and major procedures were observed to be over eight times more likely to require an unplanned post-operative ICU admission if they consumed more than $72 \mathrm{~g}$ of alcohol per day [14]. Trauma patients who consume alcohol in excess are more likely to experience a range of morbidities including pancreatitis, pneumonia, bleeding requiring transfusion and longer ICU stay [15]. Burns patients who consume alcohol excessively are more likely to die [16].

Hence, in addition to those ICU admissions which were measured in this study, there exists another cohort of patients described above who consume alcohol excessively, experience greater morbidity as a consequnce of this, and are more likely to have an ICU admission, even if their admission diagnosis is not one of those which formed the inclusion criteria for this study. In this regard, it is possible that whilst our study captured those ICU admissions directly related to alcohol misuse, it may underestimate the actual burden of alcohol-related ICU workload.

We did not define alcohol misuse in this study in relation to an absolute level of alcohol consumption. Various definitions exist in this regard, the common feature of which is excessive alcohol intake causing harm, and this concept was used, together with the admission diagnoses to capture the study group. Given the very heterogenous and inter-individual response to both acute and chronic alcohol consumption, it was felt that definitions based on quantity of alcohol consumed per unit time would neither add meaningfully to the study nor change the number of patients meeting the inclusion criteria. Furthermore, measuring blood alcohol level in this setting does not generally influence clinical decision making and we did not do so unless specifically indicated.

This study has strengths; the data was prospectively collected using clear definitions that describe an unequivocal relationship between alcohol misuse and diagnosis. Study limitations include observation of a discrete time period in one centre. It is difficult to assess the applicability of our findings to other institutions; a larger multi-centre investigation would indicate the burden of alcohol-related illness on the wider Irish ICU system.

\section{Conclusion}

This study, the first of its kind in the Irish setting, suggests that previous assessments of alcohol-related ICU workload may have underestimated the scale of the problem. Approximately one-sixth of available ICU bed-days during the study period were occupied as a result of alcoholrelated illness. Significant ICU resources are being consumed which is having an impact on the ability of the ICU to support other hospital services and elective surgical procedures. Our findings give further insight into the scale of the healthcare-related problems associated with alcohol misuse and we suggest prioritisation of primary preventative strategies in relation to this treatable problem.

\section{References}

1. Health Service Executive Alcohol Implementation Group (2008) Alcohol-related harm in Ireland. Health Service Executive, Dublin

2. Fernandez-Sola J, Junque A, Estruch R, Monforte R, Torres A, Urbano-Marquez A (1995) High alcohol intake as a risk and prognostic factor for community-acquired pneumonia. Arch Intern Med 155(15):1649-1654

3. Bercault N, Boulain T (2001) Mortality rate attributable to ventilator-associated nosocomial pneumonia in an adult intensive care unit: a prospective case-control study. Crit Care Med 29(12):2303-2309

4. Moss M, Bucher B, Moore FA, Moore EE, Parsons P (1996) The role of chronic alcohol abuse in the development of acute respiratory distress syndrome in adults. JAMA 275(1):50-54

5. O'Brien J, Lu B, Ali NA et al (2007) Alcohol dependence is independently associated with sepsis, shock, and hospital mortality among adult intensive care unit patients. Crit Care Med 35(2):345-350

6. World Health Organisation (2004) Global status report on alcohol 2004. World Health Organisation, Geneva

7. World Health Organisation (2009) European health for all database. http://data.euro.who.int/hfadb/

8. Department of Health and Children (2004) Strategic task force on alcohol second report. Department of Health and Children, Dublin 
9. Mongan D, Reynolds S, Fanagan S, Long J (2007) Health-related consequences of problem alcohol use. Health Research Board, Dublin

10. Martin J (2009) Alcohol-attributable fractions for ireland-attributable deaths and hospitalisations. Proceedings of the faculty of public health medicine royal college of physicians of Ireland, Summer scientific meeting, May 26-27, Dublin, Ireland. RCPI, Dublin

11. Health Service Executive (2009) National hospitals office performance unit. http://www.hse.ie/eng/HSE_FactFile/County_ Information/Dublin/St\%20James\%20Hospital\%20Dublin/St_ James_Hospital_Dublin.html

12. Uusaro A, Parviainen I, Tenhunen JJ, Ruokonen E (2005) The proportion of intensive care unit admissions related to alcohol use: a prospective cohort study. Acta Anesthesiol Scand 49:1236-1240
13. Trainor D, Martin L, Lavery G (2009) Alcohol misuse requiring admission to a tertiary referral ICU: resource implications. Crit Care 13(Suppl 1):p461

14. Delgado-Rodriguez M, Gomez-Artego A, Mariscal-Ortiz M, Palma-Perez S, Sillero-Arenas M (2003) Alcohol drinking as a predictor of intensive care and hospital mortality in general surgery: a prospective study. Addiction 98:611-616

15. Jurkovich J, Rivara FP, Gurney JG et al (1993) The effect of acute alcohol intoxication and chronic abuse on outcome from trauma. JAMA 270(1):51-56

16. McGill V, Kowal-Vern A, Fisher SG, Kahn S, Gamelli RL (1995) The impact of substance abuse on mortality and morbidity from thermal injury. J Trauma 38:931-934 